Song et al., Afr J Tradit Complement Altern Med., (2017) 14 (4): 8-21

https://doi.org/10.21010/ajtcam.v14i4.2

\title{
RAW REHMANNIA RADIX POLYSACCHARIDE CAN EFFECTIVELY RELEASE PEROXIDATIVE INJURY INDUCED BY DUCK HEPATITIS A VIRUS
}

\section{Meiyun Song, Yun Chen, Hongxu Du, Shuaibing Zhang, Yixuan Wang, Ling Zeng, Jingjing Yang Jintong Shi, Yi Wu, Deyun Wang, Yuanliang Hu, Jiaguo Liu*}

\author{
Institute of Traditional Chinese Veterinary Medicine,College of Veterinary Medicine,
}

Nanjing Agricultural University, Nanjing 210095, P R China.

*Corresponding author E-mail: liujiaguo@njau.edu.cn

\begin{abstract}
Background: Duck viral hepatitis (DVH), caused by duck hepatitis A virus (DHAV), is a fatal contagious infectious disease which spreads rapidly with high morbidity and high mortality, and there is no effective clinical drug against DVH.

Materials and Methods: Raw Rehmannia Radix Polysaccharide (RRRP), Lycii Fructus polysaccharides and Astragalus Radix polysaccharides were experimented in vitro and in vivo. Mortality rate, livers change, liver lesion scoring, peroxidative injury evaluation indexes in vitro and in vivo, and hepatic injury evaluation indexes of optimal one were detected and observed in this experiment.

Results: RRRP could reduce mortality with the protection rate about $20.0 \%$ compared with that of the viral control (VC) group, finding that RRRP was the most effective against DHAV. The average liver scoring of the VC, blank control (BC), RRRP groups were 3.5, 0, 2.1. Significant difference $(P<0.05)$ appeared between any two groups, demonstrating that it can alleviate liver pathological change. RRRP could make the hepatic injury evaluation indexes similar to BC group while the levels of the VC group were higher than other two groups in general. The levels of SOD, GSH-Px, CAT of RRRP group showed significant higher than that of VC group while the levels of NOS and MDA showed the opposite tendency, thus, RRRP could release peroxidative injury.

Conclusion: RRRP was the most effective against duck hepatitis A virus (DHAV). RRRP could reduce mortality, alleviate liver pathological change, down-regulate liver lesion score, release peroxidative injury and hepatic injury. The antiviral and peroxidative injury releasing activity of RRRP for DHAV provided a platform to test novel drug strategies for hepatitis A virus in human beings.
\end{abstract}

Key words: Raw Rehmannia Radix Polysaccharide; duck hepatitis A virus; peroxidative injury; hepatic injury.

Abbreviations: DHAV, duck hepatitis A virus; DVH, Duck viral hepatitis; DHV, duck hepatitis virus; RRRP, Raw Rehmannia Radix Polysaccharide; LFP, Lycii Fructus polysaccharides; ARP, Astragalus Radix polysaccharides; DMEM, Dulbecco's modified Eagle's medium; MM, maintain medium; D-Hank's, Dulbecco's Hanks balanced salt solution; PBS, magnesium-free phosphate-buffered saline; MTT, 3-(4, 5-dimethylthiazol-2-yl)-2, 5-diphenyltetrazolium bromide; SOD, superoxide dismutase; GSH-PX, glutathione peroxidase; CAT, catalase; MDA, malondialdehyde; NOS, nitric oxide synthase; TAC, total antioxidant capacity; DEHs, duck embryonic hepatocytes; VIR, inhibitory rate of virus; CC,cell control; VC, virus control; BC, blank control; NS, normal saline; ALT, alanine aminotransferase; AST, aspartate transaminase; ALP, alkaline phosphatase. 


\section{Song et al., Afr J Tradit Complement Altern Med., (2017) 14 (4): 8-21 \\ https://doi.org/10.21010/ajtcam.v14i4.2}

\section{Introduction}

Duck viral hepatitis (DVH) caused by duck hepatitis virus (DHV), is a fatal contagious infectious disease which spreads rapidly with high morbidity and high mortality (Wen et al., 2014). Furthermore, duck viral hepatitis type A is the perfect animal model of viral hepatitis type A in human beings. The proofs are illustrated as below. On the basis of virus classification, duck hepatitis A virus and hepatitis A virus both belong to Picornaviridae. Hepatitis A virus is only member of the Genus Hepatovirus causing human infection (Singh et al., 2015) while duck hepatitis A virus is a member of Genus Avihepatovirus (Erfan et al., 2015). Therefore, duck hepatitis A virus is similar to hepatitis A virus to some degree. On the other hand, children are vulnerable to be infected by hepatitis A virus (Triassi et al., 2012) while ducklings, under the age of 21 day, are those most easily to be effected by duck hepatitis A virus (Wen et al., 2014).

DHV has three serotypes which have apparent diversity and have no cross-immunity. Researchers have confirmed that DHAV is prevalent in China (Fu et al., 2008; Shi et al., 2013; Wei et al., 2012). The circumstance of the two diseases share similarity in the area of liver lesion, mental status, temperature change and so on. There are few studies in animal models using modern methodologies for viral hepatitis type A in human beings (Lanford et al., 2011).

There is no effective clinical drug against DVH while the prevention of DVH is vaccine, yolk antibody and monoclonal antibody (Kim et al., 2009). The high price, inconvenience to store and transport make the prevention and treatment difficult. Once infected, a large number of ducks will die because of the lack of effective drugs.

More and more polysaccharides and their sulfating showed the same capacity of antivirus (García-Villalón et al., 1991; Lee et al., 2002; Shen et al., 2004), including anti-DHAV. Yun Chen et al. did the research that Bush Sophora Root polysaccharide and its sulfate were effective to anti-DHAV both in vitro and vivo by scavenging free radicals in vivo (Chen et al., 2014a, b). Raw Rehmannia Radix polysaccharides (RRRP), Lycii Fructus polysaccharides (LFP) and Astragalus Radix polysaccharides (ARP) are common ones against viral disease (Li et al., 2014; Kim et al., 2011; Li et al., 2013; Cui et al., 2012; Li et al., 2010). LFP exhibited remarkable protective effects against diethylnitrosamine-induced oxidative hepatic injury in liver cancer rats (Cui et al., 2012). The antioxidant potential of Astragalus was evaluated by using the DPPH test, finding that it was principally responsible for the radical-scavenging activity (Olszewska et al., 2012). Researchers have also found that RRRP had antioxidative activity on Hep G2 cells and in mice (Nuong et al., 2014; Sui et al., 2013).

Free radicals play a role in the pathogenesis of liver damage (Cardin et al., 2002). Ferritin levels were positively correlated with the amount of malondialdehyde (MDA) (Livrea et al., 1996). The therapeutics of hepatitis is intimately related to degree and quality of the immune response and the antioxidant capacity (Strick-Marchand et al., 2015; Luper, 1998). As for DVH, study has proved that the oxidative stress plays an important role in the pathogenesis (Xiong et al., 2014). The pharmacal antioxidant effect might alleviate the hepatic injury induced by DHAV (Chen et al., 2014b).

Raw Rehmannia Radix, Lycium Fructus and Astragalus, belonging to traditional Chinese drugs, are often used in clinic. Lots of researches certify polysaccharides are the key effective components. They are widely used in prevention and treatment in RNA viral disease. People have investigated that sulfated Chuanmingshen violaceum polysaccharide, astragalus polysaccharide and sulfated epimedium polysaccharide had activities against Newcastle disease virus (Song et al., 2013; Guo et al., 2012). Edible mushroom Pleurotus ostreatus and sulfated Astragalus polysaccharide could resist infectious bursal virus (Huang et al., 2008; Selegean et al., 2009). Study suggested that the polysaccharides obtained from Azadirachta indica act against poliovirus-1 by inhibiting the initial stage of viral replication (Faccin-Galhardi et al., 2012). On account of above mentioned and to develop effective clinical drug to anti-DVH, the anti-DHAV effects of RRRP, LFP and ARP to anti-DHAV in vitro and in vivo are compared. The peroxidative injury releasing ability of the optimization (RRRP) was observed in vitro and in vivo. 


\section{Materials and methods}

Reagents, virus, duck embryos and ducklings

Dulbecco's modified Eagle's medium (DMEM, Gibco) containing penicillin $100 \mathrm{IU} / \mathrm{mL}$, streptomycin $100 \mathrm{IU} / \mathrm{mL}$, $10 \%$ fetal bovine serum and glutamine $0.75 \mathrm{mg} / \mathrm{mL}$ was provided to cultivate the duck embryonic hepatocytes. Maintain medium (MM) was the same with DMEM except that fetal bovine serum was $1 \%$. Dulbecco's Hanks balanced salt solution (D-Hank's) acted as cleanout fluid for the embryo tissue fragments and cells. Trypsin (Amresco) was dissolved into $0.20 \%$ with D-Hank's. The 3-(4, 5-dimethylthiazol-2-yl)-2, 5-diphenyltetrazolium bromide (MTT, Amresco) was dissolved with calcium and magnesium-free phosphate-buffered saline (PBS) into $5 \mathrm{mg} / \mathrm{mL}$. Heparin sodium (Amresco) was dissolved into $2 \mathrm{mg} / \mathrm{mL}$ with physiological saline. Trypsin, MTT and Heparin sodium solutions were filtered through $0.22 \mu \mathrm{m}$ syringe filters. DMEM was stored at $4{ }^{\circ} \mathrm{C}$ and MTT solution was stored at $4{ }^{\circ} \mathrm{C}$ in brown bottles. Other chemicals used in the experiment were analytical grade.

Duck superoxide dismutase (SOD) ELISA kit (Lot no. 20141211), glutathione peroxidase (GSH-PX) ELISA kit (Lot no. 20141215), catalase (CAT) ELISA kit(Lot no. 20141211), MDA ELISA kit(Lot no. 20141211), nitric oxide synthase (NOS) ELISA kit(Lot no. 20141217), total antioxidant capacity (TAC) ELISA kit(Lot no. 20141216 ) were bought from Jiancheng Biotechnology Co., Ltd.

DHAV ( $L Q_{2}$ strain), whose $\mathrm{TCID}_{50}$ indicated $1 \times 10^{-3}$, was supplied by the Shandong Institute of Poultry in China and was diluted with $\mathrm{MM}$ into $5 \times 10^{-2}\left(50 \mathrm{TCID}_{50}\right)$ for the following tests.

Duck embryos were bought from Nanjing Institute of Animal Husbandry and Poultry Science. The 4-day-old cherry valley Ducklings were purchased from Tangquan Poultry Farm, Jiangsu province, China.

All animal experiments were carried out in accordance with the guidelines set forth by the Institutional Animal Care and Use Committee (IACUC), Nanjing Agricultural University IACUC.

\section{Preparation of RRRP, LFP and ARP}

The method of the extract of RRRP was described as Sui (Sui et al., 2013). 1000g of smashed radix rehmanniae (bought from Jingui Chinese herb yinpian Co.,Ltd, Hubei, China, Lot no. A40805) was decocted with 6-fold water for 2 $\mathrm{h}$ for the first time. After filtration, the residue was boiled again with 6 -fold water for $2 \mathrm{~h}$. The colatuie above was concentrated to small volume and became supernatant through centrifugation. $95 \%$ ethanol was added into the water-extraction to make ethanol concentration to $75 \% .24 \mathrm{~h}$ later, the precipitation was stored at $-20^{\circ} \mathrm{Cfor} 6 \mathrm{~h} .234 .03 \mathrm{~g}$ was acquired after lyophilization. Its final polysaccharide content suggested to $84.5 \%$ via phenol-sulfuric acid method (Wang et al., 2015).

LFP (Lot no.20140928) and ARP (Lot no. 20140928) were bought from Shanxi Ciyuan Biotechnology Co.,Ltd with final polysaccharide content of $83.5 \%$ and $75.0 \%$, respectively.

\section{The preparation of the duck embryonic hepatocytes (DEHs)}

The steps followed the method of Chen (Chen et al., 2014a). Livers were aseptically removed from duck embryonic with eliminating gallbladder and then were cut into pieces. In order to remove the blood, D-Hank's was used to wash livers three times both before and after mashing them. Trypsin digestion for 5 minutes was determined to obtain single duck embryonic hepatocyte. After the cells were washed with D-Hank's for 3 times, DMEM appeared on the stage to culture them. The final cell concentration should reach to $0.8 \times 10^{6}-1.2 \times 10^{6} \mathrm{~mL}^{-1}$ and the DEHs were cultivated at $37^{\circ} \mathrm{C}$ in a $5 \%$ humidified $\mathrm{CO}_{2}$ incubator. The growth medium replaced after $24 \mathrm{~h}$ until the cells became monolayer. 
Song et al., Afr J Tradit Complement Altern Med., (2017) 14 (4): 8-21

https://doi.org/10.21010/ajtcam.v14i4.2

\section{Comparison of anti-DHAV activity of RRRP, LFP and ARP in vitro and in vivo The anti-DHAV activity of RRRP, LFP and ARP in vitro}

$100 \mu \mathrm{L} 50 \mathrm{TCID}_{50} \mathrm{DHAV}$ was put in the 96 -well plate, then removed after cultivated at $37{ }^{\circ} \mathrm{C}$ in a $5 \%$ humidified $\mathrm{CO}_{2}$ incubator for $2 \mathrm{~h}$. The D-hanks was used to wash the 96-well plate two times. The drug was treated by multiple proportion dilution while the initial concentration was the maximum safe concentration. $100 \mu \mathrm{L}$ liquid was put into every well and every dilution contained 4 repetitions. Meanwhile, virus control (VC) and cell control (CC) groups should be set. The 96 -well plate was cultivated at $37^{\circ} \mathrm{C}$ in a $5 \%$ humidified $\mathrm{CO}_{2}$ incubator for $96 \mathrm{~h}$ before the $A_{570}$ value was detected by MTT method (Emzhik et al., 2015). At the same time, the virus inhibitory rate (VIR) was according to the formula: $V I R=\left(\bar{A}_{\text {drug }+ \text { virus }}-\bar{A}_{\mathrm{VC}}\right) /\left(\bar{A}_{\mathrm{CC}}-\bar{A}_{\mathrm{VC}}\right) \times 100 \%$. The antiviral effect referred to $A_{570}$ and VIR while the effective antiviral concentration was the dilution whose $A_{570}$ value was significantly higher than that of the VC group.

\section{The anti-DHAV activity of RRRP, LFP and ARP in vivo}

There were 5 groups, including VC group, blank control (BC) group and other three drug groups (RRRP, LFP and ARP groups). Each group possessed 45 feathers 4-day-old cherry valley ducklings. 180 ducklings were intramuscular injected with $5 \mathrm{LD}_{50}$ DHAV and randomly divided into 4 groups, including the VC, RRRP, LFP, and ARP groups.

While the residual ducklings served as the BC group (isolated reared) were intramuscular injected with normal saline (NS) with the same volume. Two hours later, the ducklings of in the RRRP, LFP and ARP groups were got $3 \mathrm{mg}$ net drug per duck through drinking water, once a day for consecutive 5 days. However, ducklings in the VC and BC groups were just provided with the normal water at the same time. The feathers of dead ones were recorded. The treatment effect of drugs was valued by mortality rate (Mortality rate=deaths $/$ sample $\times 100 \%$ ).

\section{The assay for antioxidant capacity of RRRP in vitro and in vivo}

Dynamic deaths and livers lesion scoring

The methods of animal grouping and treatment were the same as 2.4.2. In brief, 150 4-day-old cherry valley ducklings were randomly divided into 3 groups on average which were the RRRP group, VC group and BC group.

The ducklings of the RRRP and VC groups were intramuscular injected with $5 \mathrm{LD}_{50}$ DHAV while the ducklings of the $\mathrm{BC}$ group were intramuscular injected with NS with the same volume. The ducklings of the RRRP group were got $3 \mathrm{mg}$ net drug per feather through drinking water as above. The ducklings in the $\mathrm{BC}$ group were raised separately from the other two groups. The anticoagulated blood was kindly collected at the time of 4th $\mathrm{h}, 8$ th $\mathrm{h}$ and 54th $\mathrm{h}$ with the addition of heparin sodium after inoculation with DHAV. Five ducklings in each group were individually stored for blood sampling at each stage. Observed the clinical symptoms and dynamic deaths every day and recorded the deaths of each group at the periods of $12 \mathrm{~h}, 24 \mathrm{~h}, 30 \mathrm{~h}, 36 \mathrm{~h}, 48 \mathrm{~h}, 72 \mathrm{~h}, 96 \mathrm{~h}$ and $120 \mathrm{~h}$. The dead ones were timely dissected and their livers' lesion was scored according to the standard. According to the degree of liver pathological change, the livers scored ranging from 0 to 5 . The hepatic lesions were assessed by average liver lesion score $\left\{\sum\right.$ (different scoring $\times$ number)/sample number\}. When the ducklings stopped dying, the statistics of mortality of each group should be analyzed. In the end, the effect of RRRP anti-DVH was estimated by visual change, mortality and average liver lesion score. Liver lesion scoring criteria(Cardozo et al., 2013): 0 represented the livers of the ducklings alive; 1 stood for either livers with less than 10 hemorrhages or not more than 1 ecchymosis; 2 conformed to the color of liver was normal on the whole with 10-100 hemorrhages; 3 was that the liver turned red slightly and a third area of liver was filled with haemorrhages; 4 points demonstrated that $1 / 2$ area of liver appeared hemorrhages with the liver already 
Song et al., Afr J Tradit Complement Altern Med., (2017) 14 (4): 8-21

https://doi.org/10.21010/ajtcam.v14i4.2

became red on the large scale; 5 corresponded to the situation that the liver was covered with diffuse hemorrhages with the liver developing into dark red and there were multiple ecchymoses.

\section{Evaluation indexes of hepatic injury and peroxidative injury in vivo and in vitro \\ Evaluation indexes of hepatic injury in vivo}

The serum levels of alanine aminotransferase (ALT), aspartate transaminase (AST), alkaline phosphatase (ALP) at 4th, 8th and 54th h were tested by automatic biochemistry analyzer (7180 Automatic Analyzer, HITACHI, Japan) in Nanjing Ao Qing Bio-Tech Co.,Ltd.

\section{Evaluation indexes of peroxidative injury in vivo}

After centrifugation of $3500 \mathrm{rpm} / \mathrm{min} 10 \mathrm{~min}$, the activity of SOD, CAT, GSH-PX, NOS and the level of MDA of plasma were determined under the directions of the Duck SOD ELISA kit, GSH-PX ELISA kit, CAT ELISA kit, MDA ELISA kit and NOS ELISA kit.

\section{Evaluation indexes of peroxidative injury in vitro}

The DEHs were set into 3 groups including the VC, CC and RRRP groups when the DEHs became monolayer.

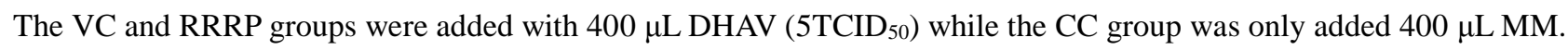
The 6-well plates were cultivated at $37{ }^{\circ} \mathrm{C}$ in a $5 \%$ humidified $\mathrm{CO}_{2}$ incubator for $2 \mathrm{~h}$. The most effective concentration of RRRP was added into the DEHs in the plate. Each group enjoyed 3 replications. The 6-well plates were cultivated at $37{ }^{\circ} \mathrm{C}$ in a $5 \%$ humidified $\mathrm{CO}_{2}$ incubator for $24 \mathrm{~h}$. The DEHs were twice washed by PBS before they were scraped. 0.6 $\mathrm{mL}$ PBS was added to the well to make the cell density up to $3 \times 10^{5}-5 \times 10^{5} / \mathrm{mL}$. DEHs in the EP tubes were crushed with the ultrasonic cell disruptor (13-0528, Ningbo Xinzhi Bio-Tech Co.Ltd.). Make sure that the probe was soakaged into liquid. The power of ultrasonication was 300W, 3-5s per time with four intervals. The cells were in the ice water all over the process of ultrasonication. The activities of SOD, CAT, GSH-PX, NOS, TAC and the level of MDA of cell disruption liquor were detected. The approach to evaluate them was according to the introduction of Duck SOD ELISA kit, GSH-PX ELISA kit, CAT ELISA kit, MDA ELISA kit, NOS ELISA kit and TAC ELISA kit.

The correlation analysis of mortality rate, hepatic injury evaluation indexes and peroxidative injury evaluation indexes

Mortality rate of the VC, BC and RRRP groups at the time of 54th $\mathrm{h}$ after inoculation with DHAV were calculated. Hepatic injury evaluation indexes and peroxidative injury evaluation indexes at the same time were ready for the following analysis. The correlation analysis of mortality rate, evaluation indexes of hepatic injury and peroxidative injury were done with the method of Pearson coefficient by SPSS Software Package v 19.0.

\section{Statistical analysis}

The software of SPSS Package v 19.0 was applied to the Statistical analysis. The form of mean \pm SD was utilized for the data of anti-DHAV activity, evaluation indexes of peroxidative injury and hepatic injury. Duncan's multiple analysis was used for the comparison of the distinction between each group. Mortality was analyzed by chi square test. Statistical significance was assumed at $p<0.05$. 
Song et al., Afr J Tradit Complement Altern Med., (2017) 14 (4): 8-21

https://doi.org/10.21010/ajtcam.v14i4.2

\section{Results}

\section{Comparison of anti-DHAV activity of RRRP, LFP and ARP in vitro and in vivo}

\section{The anti-DHAV activity in vitro}

Table 1 told $A_{570}$ values and VIR of RRRP, LFP and ARP in vitro on DEHs. As the concentration changed, $A_{570}$ and VIR also altered. $A_{570}$ of the RRRP group except for maximum concentration, medial two concentrations of the LFP group, and at the concentration of $156.250 \mu \mathrm{g} \cdot \mathrm{mL}^{-1}$ of the ARP group were significantly higher than that of the VC group. The homologue topmost VIR of RRRP, LFP and ARP were 32.6\%, 61.0\% and 35.2\%, respectively.

Table 1: $A_{570}$ value and VIR of RRRP, LFP and ARP groups in vitro to anti- DHAV on DEHs

\begin{tabular}{|c|c|c|c|c|c|c|c|}
\hline \multirow{2}{*}{ Treatment } & \multirow{2}{*}{$\begin{array}{l}\text { Concentration } \\
/ \mu \mathrm{g} \cdot \mathrm{mL}^{-1}\end{array}$} & \multicolumn{2}{|l|}{ RRRP group } & \multicolumn{2}{|l|}{ LFP group } & \multicolumn{2}{|l|}{ ARP group } \\
\hline & & $A_{570}$ & $V I R / \%$ & $A_{570}$ & $V I R / \%$ & $A_{570}$ & $V I R / \%$ \\
\hline \multirow{4}{*}{ Drugs } & 625.000 & $0.448 \pm 0.015^{\mathrm{cd}}$ & 10.9 & $0.388 \pm 0.021^{\mathrm{cd}}$ & 4.5 & $0.398 \pm 0.012^{\mathrm{bc}}$ & 10.4 \\
\hline & 312.500 & $0.473 \pm 0.024^{\mathrm{bc}}$ & 18.9 & $0.578 \pm 0.013^{\mathrm{b}}$ & 61.0 & $0.420 \pm 0.009^{b c}$ & 22.5 \\
\hline & 156.250 & $0.516 \pm 0.019^{b}$ & 32.6 & $0.446 \pm 0.017^{\mathrm{c}}$ & 21.7 & $0.443 \pm 0.017^{\mathrm{b}}$ & 35.2 \\
\hline & 78.125 & $0.478 \pm 0.023^{\mathrm{bc}}$ & 20.4 & $0.426 \pm 0.020^{\mathrm{cd}}$ & 15.8 & $0.414 \pm 0.004^{\mathrm{bc}}$ & 19.2 \\
\hline $\mathrm{CC}$ & & $0.727 \pm 0.010^{\mathrm{a}}$ & & $0.709 \pm 0.033^{\mathrm{a}}$ & & $0.561 \pm 0.010^{\mathrm{a}}$ & \\
\hline $\mathrm{VC}$ & & $0.414 \pm 0.008^{d}$ & & $0.373 \pm 0.03^{\mathrm{d}}$ & & $0.379 \pm 0.011^{\mathrm{c}}$ & \\
\hline
\end{tabular}

Note: Column data without same superscripts are differ significantly $(P<0.05)$.

The anti-DHAV activity in vivo

The mortality rate of the RRRP, LFP, ARP, VC and BC groups were $73.3 \%, 80.0 \%, 82.2 \%, 91.1 \%, 0 \%$, respectively. The mortality rate of the LFP group, ARP group and VC groups showed no significance while the significance appeared between the RRRP group and the VC group.

\section{Results of peroxidative injury evaluation indexes of RRRP in vitro and in vivo}

The effect of RRRP on dynamic deaths and liver lesion scoring of DHAV infected ducklings

The mortality rate of the RRRP, BC, VC groups were $71.4 \%, 0$, and $91.4 \%$, respectively. RRRP group could significantly down-regulate the deaths, resulting from the protection rate of RRRP up to $20.0 \%$ compared with that of the VC group.

Dynamic deaths and livers change of the BC, VC and RRRP groups were displayed in the Figure 1. Figure 1( I ) pictured dynamic deaths and Figure 1( II ) Figured the livers changes of the BC, VC and RRRP groups. The dynamic deaths of the VC and RRRP groups were similar in general. The death peak time of the two groups was both at $24 \mathrm{~h}$ while 16 ducklings were dead at this period, respectively. When it turned $30 \mathrm{~h}$, the deaths were sharply decreased to 4 in the VC group and 3 in the RRRP group. At the period of 48th h, there was another tiny peak time at which the VC group and RRRP group got the deaths of 5 and 2. The death of ducklings became stable at the remaining time except 5 ducklings in the VC group and 3 ducklings in the RRRP group had been dead. The livers change of different groups was showed in the right of Figure 1. The livers of the VC group were covered with diffuse hemorrhages with the color changing overall darkled and there were many ecchymosed. As for the BC group, the livers were the normal. The RRRP group's livers were equal to point 3. The livers lesion of RRRP was relieved compared to that of the VC group. 


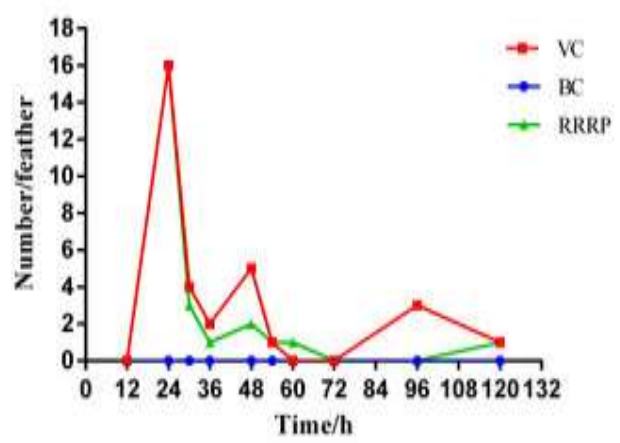

I Dynamic deaths of VC, BC, RRRP groups

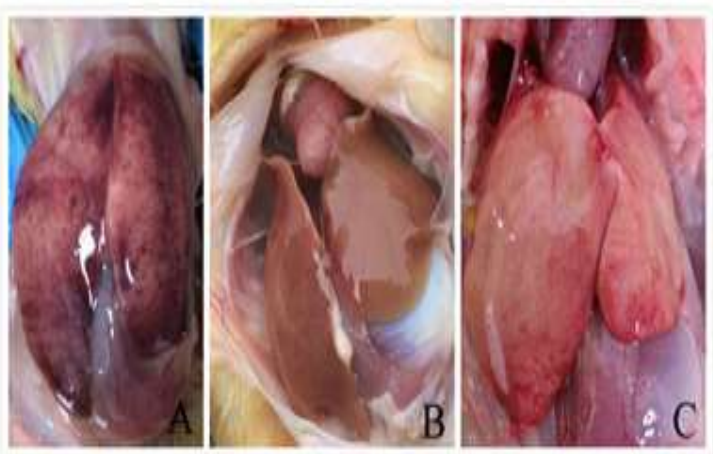

II Liver change of VC(A), BC(B), RRRP(C) groups

Figure 1: Dynamic deaths and livers change of the RRRP, BC and VC groups in vivo.

Table 2 denoted the distribution of livers lesion score of the VC, BC and RRRP groups. The score of the VC group focused on 3, 4 and 5 but the score of the RRRP group centralized to 0, 2 and 3. The distribution of the score told the information that RRRP could ease the liver lesion of the infected ducklings. The average scoring of the VC, BC, RRRP groups were $3.5,0,2.1$. Significant difference $(p<0.05)$ appeared between any two groups. The livers lesion scoring was suit for the data of duckling mortality of RRRP to anti- DHAV in vivo.

Table 2: The distribution of liver lesion score of the VC, BC and RRRP groups

\begin{tabular}{llllllll}
\hline Treatment & \multicolumn{3}{l}{ Score of liver lesion } & \multicolumn{3}{c}{ Average score } \\
& 0 & 1 & 2 & 3 & 4 & 5 & \\
\hline VC/feathers & 3 & 0 & 2 & 9 & 15 & 6 & $3.5^{\mathrm{a}}$ \\
BC/feathers & 35 & 0 & 0 & 0 & 0 & 0 & $0^{\mathrm{c}}$ \\
PRRP/feathers & 10 & 1 & 7 & 11 & 5 & 1 & $2.1^{\mathrm{b}}$ \\
\hline
\end{tabular}

Note: Row data without same superscripts are differ significantly $(P<0.05)$.

\section{The effect of RRRP on plasma biochemical evaluation indexes of hepatic injury of DHAV infected ducklings}

The results of plasma biochemical evaluation indexes of hepatic injury were illustrated in the Table 3 . The ALT level of the three groups had no significant difference at the time of 4 th $\mathrm{h}$ and $54 \mathrm{th} \mathrm{h}$. At the period of 8th $\mathrm{h}$, the ALT level of the VC group was significantly higher than that of other two groups. At the initial period of 4 th $\mathrm{h}$ and at the later period of 54th h, the VC group got a higher level of AST compared with the RRRP and BC groups. At the mid time of 8 th $\mathrm{h}$, the three groups shared no significant difference. Obviously, the levels of ALP of the VC group were higher than other two groups in all time.

Table 3: Plasma biochemical evaluation indexes of hepatic injury

\begin{tabular}{lllll}
\hline Index & Time $(\mathrm{h})$ & VC group & BC group & RRRP group \\
\hline \multirow{2}{*}{ ALT(IU/L) } & 4 & $25.3 \pm 1.5^{\mathrm{a}}$ & $29.3 \pm 2.8^{\mathrm{a}}$ & $29.5 \pm 2.4^{\mathrm{a}}$ \\
& 8 & $44.3 \pm 6.3^{\mathrm{a}}$ & $28.0 \pm 2.7^{\mathrm{b}}$ & $22.4 \pm 0.7^{\mathrm{b}}$ \\
& 54 & $47.0 \pm 3.9^{\mathrm{a}}$ & $42.3 \pm 6.3^{\mathrm{a}}$ & $39.0 \pm 1.8^{\mathrm{a}}$ \\
AST(IU/L) & 4 & $36.5 \pm 5.1^{\mathrm{a}}$ & $22.0 \pm 1.8^{\mathrm{b}}$ & $22.3 \pm 2.3^{\mathrm{b}}$ \\
& 8 & $18.7 \pm 2.7^{\mathrm{a}}$ & $18.3 \pm 1.9^{\mathrm{a}}$ & $23.0 \pm 1.0^{\mathrm{a}}$ \\
& 54 & $21.8 \pm 2.6^{\mathrm{a}}$ & $13.8 \pm 1.3^{\mathrm{b}}$ & $14.7 \pm 0.7^{\mathrm{b}}$ \\
ALP(IU/L) & 4 & $1026.7 \pm 57.9^{\mathrm{a}}$ & $639.5 \pm 33.3^{\mathrm{b}}$ & $677.0 \pm 47.4^{\mathrm{b}}$ \\
& 8 & $878.7 \pm 107.3^{\mathrm{a}}$ & $589.6 \pm 14.9^{\mathrm{b}}$ & $680.8 \pm 41.2^{\mathrm{b}}$ \\
\hline
\end{tabular}

Note: Row data without same superscripts are differ significantly $(P<0.05)$. 


\section{The effect of RRRP on plasma evaluation indexes of oxidative injury of DHAV infected ducklings}

Evaluation indexes of plasma oxidative injury were displayed in the Figure 2. The VC and RRRP groups shared significant difference at all time and in every index while the $\mathrm{VC}$ and BC groups also had significant difference in general.

The capacity of GSH-PX of the RRRP group was significantly higher than that of the VC group but the significance level was the same to that of the $\mathrm{BC}$ group both at two periods.

At the period of 8th h, SOD levels of the RRRP and BC groups were both significantly higher than that of the VC group but there was no significant difference between the two groups. When it was 54th h, the RRRP group was significantly higher than other two groups while the BC group and the VC group shared no significant difference.

NOS value of the VC group represented a high level compared with other two groups at 8 th $\mathrm{h}(P<0.05)$. At the time of 54th h, the VC group was discrepantly higher than the BC group while the BC group was discrepantly higher than the RRRP group.

CAT levels of the three groups were separated into two sides. On the one hand, CAT level of the RRRP group was discrepantly higher than that of the VC group both at 8th h and 54th h. On the other hand, the VC group had no discrepantly difference with the $\mathrm{BC}$ group at 8 th $\mathrm{h}$ but the status changed at 54 th $\mathrm{h}$.

For MDA, the RRRP and BC groups were higher than the VC group at 8 th $\mathrm{h}$ with the significance level of $p<0.05$, while the RRRP group and the BC group were lower than that of the VC group at the time of 54th $\mathrm{h}$ with the significance level of $p<0.05$.
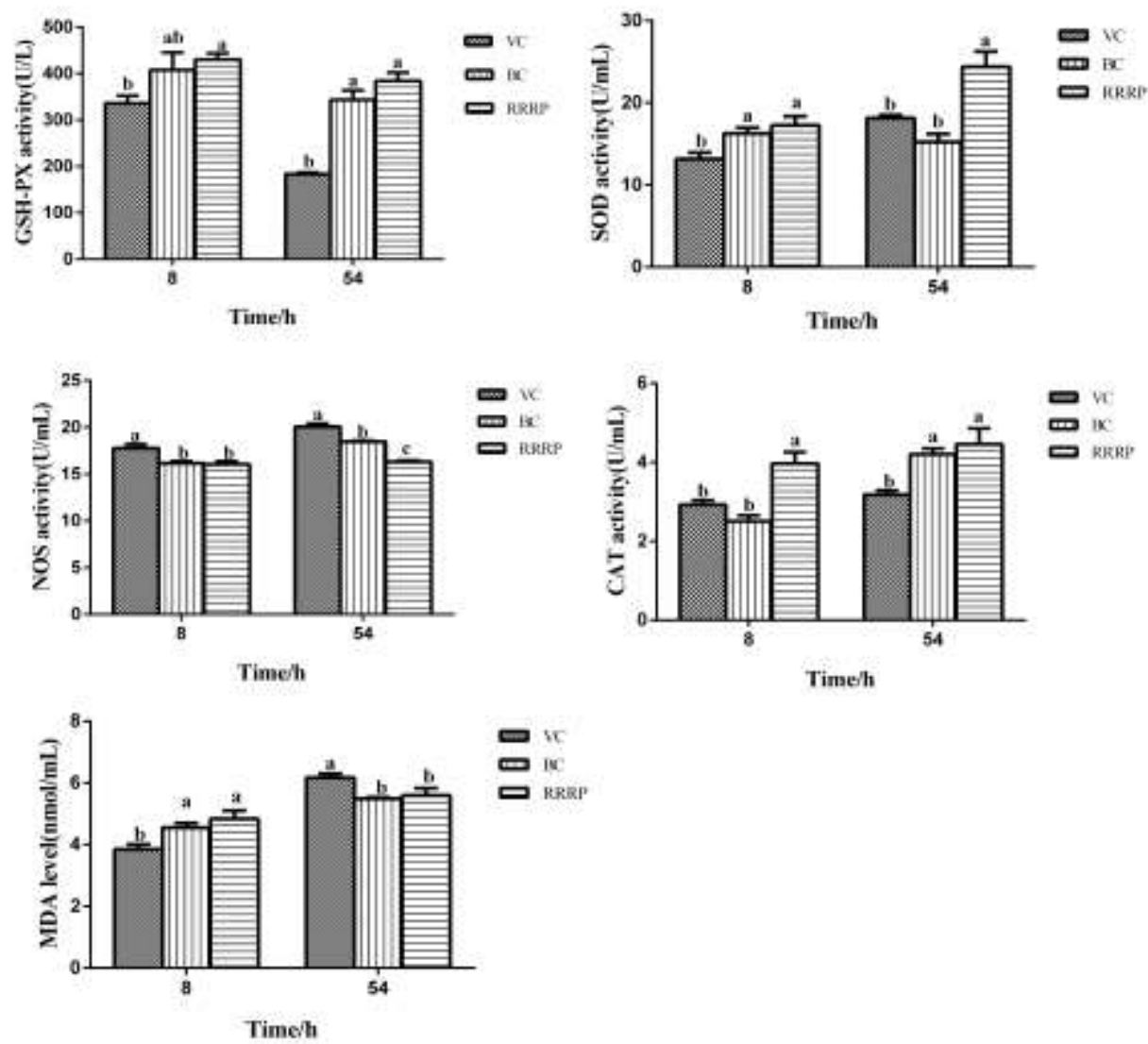

Figure 2: The activity of plasma peroxidative injury evaluation indexes Note: ${ }^{a-b}$ Bars without the same superscripts $(a-b)$ in the same time differ significantly $(P<0.05)$. 
Song et al., Afr J Tradit Complement Altern Med., (2017) 14 (4): 8-21

https://doi.org/10.21010/ajtcam.v14i4.2

The effect of RRRP on evaluation indexes of oxidative damage of DEHs induced by DHAV

Figure 3 illustrated the effect of RRRP on evaluation indexes of oxidative damage of DHAV infected DEHs. The activities of GSH-PX, SOD, CAT and TAC of the RRRP group were higher than that those of the VC group with significant difference $(p<0.05)$, while the RRRP group and the CC group shared no significant difference in these indexes except the activity of CAT. As for NOS activity, the VC group was significantly higher than the RRRP and CC groups while the RRRP group and the CC group shared no significant difference. The level of MDA suggested that the VC group was higher than that of the RRRP group and the RRRP group was higher than that of the CC group with significant difference, respectively. The activity of TAC showed that the VC group was significantly lower than that of the RRRP group while the CC group had no significant difference with other two groups $(p<0.05)$.
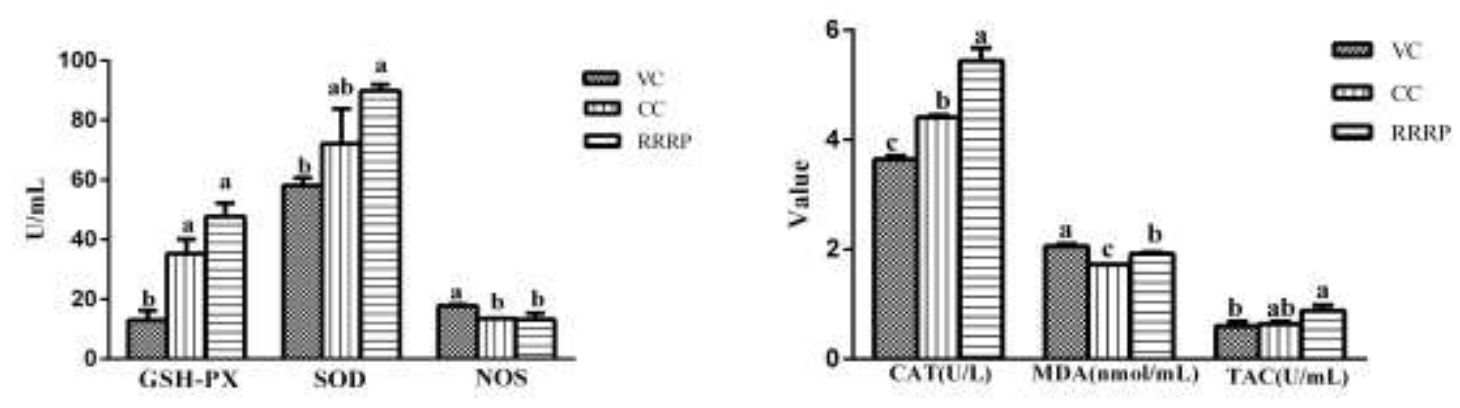

Figure 3: The activity of peroxidative injury evaluation indexes of DEHs induced by DHAV

Note: ${ }^{\mathrm{a}-\mathrm{c}}$ Bars without the same superscripts $(\mathrm{a}-\mathrm{c})$ in the same time differ significantly $(P<0.05)$.

The correlation analysis of mortality, evaluation indexes of hepatic injury and peroxidative injury

The Pearson correlation coefficients between evaluation indexes of hepatic injury, mortality and peroxidative injury were listed in the Table 4. Peroxidative injury evaluation indexes including GSH-PX, SOD, CAT and hepatic injury evaluation indexes including ALT, AST, ALP and mortality shared a positive correlation. Peroxidative injury evaluation indexes including MDA, NOS and hepatic injury evaluation indexes including ALT, AST, ALP and mortality shared a negative correlation. Significant positive correlation was occurred between MDA and AST, ALP.

Table 4: The Pearson correlation coefficients of mortality rate, evaluation indexes of hepatic injury and peroxidative injury.

\begin{tabular}{llllll}
\hline & GSH-PX & SOD & NOS & CAT & MDA \\
\hline ALT & -0.974 & -0.590 & 0.983 & -0.973 & 0.839 \\
AST & -0.956 & -0.105 & 0.758 & -0.957 & $0.999 *$ \\
ALP & -0.975 & -0.177 & 0.804 & -0.976 & $0.992 *$ \\
Mortality rate & -0.462 & -0.636 & 0.067 & -0.464 & 0.736 \\
\hline
\end{tabular}

Note: $* P<0.05$.

\section{Discussion}

The antiviral activity of drug is accessed via vitro and vivo experiments. The VIR of the RRRP, LFP and ARP 
groups in vitro were $32.6 \%, 61.0 \%$ and $35.2 \%$, respectively (Table 1 ). On the contrast, the outcome of the therapeutic effects against DVH claimed that RRRP acted best. The therapeutic effects of LFP and ARP were slightly better than that of the VC group. Why the results in vitro and in vivo were different? Based on the theory of traditional Chinese medicine, Raw Rehmanniae Radix, Lycii Fructus and Astragalus belong to heat-removing drug, nourishing Yin drug and Qi-reinforcing agent, respectively. As excess-heat syndrome disease in this theory, the way to cure DVH is removing heat to cool blood which leads to the good therapy of RRRP. Chen Yun also found that Bush Sophora Root polysaccharide acts better than ARP to against DHAV (Chen et al., 2015). In traditional Chinese medicine theory, Bush Sophora also belongs to heat-removing one. The circumstance of Chen Yun gave another evidence for the theory.

In the view that RRRP was optimal one to against DHAV, we did the further research to investigate the therapeutic effect and the change of peroxidative injury and hepatic injury evaluation indexes during the treatment of DVH in vivo. RRRP can reduce mortality rate, liver lesion scoring, alleviate visual liver lesion, and decrease the changes of plasma biochemical evaluation indexes of hepatic injury. The fact explains that hepatic injury degree corresponds to the mortality rate and RRPP can effectively release the hepatic injury induced by DHAV.

The mechanism of hepatic injury is related to reactive metabolites formation (Bhopale et al., 2006), oxidative stress induction, intracellular targets dysfunction, and immune-mediated toxicity (Heidari et al., 2015; He et al., 2015), kupffer cell depression(Hu et al., 2015), anoxemia and anoxia(Henrion, 2012). Among them, reactive oxygen species can be an important mechanism in the pathophysiology of hepatic disorders (Gabriel et al., 1998). On the other hand, measuring antioxidants status directly in the liver tissues gives an accurate estimation about the condition of the liver (Abd Ellah, 2011). The hepatorenal toxicity could be due to induction of oxidative stress in liver (Adedara et al., 2012).

Consequently, peroxidative injury stands out in the pathophysiology of hepatic injury. The method of assessing oxidative stress in this experiment was studying enzymes, such as SOD, GSH-PX, CAT, NOS and the content of free radical, such as MDA. SOD is the most important substance to remove free radicals in vivo with specific physiological activity (Kim et al., 2015). GSH-PX could turn toxiferous GSH into innoxious GSSG, resulting in protecting membrane from interference and lesion of peroxides (Jacobson et al., 2012). The main function of CAT is to catalysis $\mathrm{H}_{2} \mathrm{O}_{2}$ into $\mathrm{H}_{2} \mathrm{O}$ and $\mathrm{O}_{2}$, so that $\mathrm{H}_{2} \mathrm{O}_{2}$ will not turn the harmful thing under the role of iron chelates. The three evaluation indexes are good for liver to get rid of free radicals such as $\mathrm{O}^{2-}, \mathrm{H}_{2} \mathrm{O}_{2}$ and $\mathrm{GSH}$ (Demir et al., 2013; Kankofer et al., 2013; Cumurcu et al., 2013), which could protect ducks from oxidation injury. SOD and CAT are the members of antioxidant chain. NOS synthesizes NO that regulates multiple cellular responses by S-nitrosylation (Zhu et al., 2015). NOS is divided into 3 types (nNOS, iNOS and eNOS). MDA is a stable end product of peroxidation of membrane lipids by free radicals and it is an indicator of increased lipid peroxidation (Khademi et al.,2014).

In this paper, the antioxidant capacities of RRRP in vitro and in vivo were conformity in general (Figure 2 and Figure 3). In vivo test, the activities of SOD, GSH-PX, CAT of the RRRP group were significantly higher than those of the VC group in all periods, which indicated good clearance of free radical of RRRP. As for the level of MDA and the activity of NOS, the VC group was significantly higher than that of the RRRP group except MDA for 8th h. The decrease of MDA and NOS levels suggest the good anti-oxidative effect. In vitro test, GSH-PX activity of the RRRP group was significantly higher than that of the VC group, as well as the activity of SOD and CAT. The activity of NOS of the RRRP group was lower than that of the VC group. The evaluation indexes in vitro and in vivo shared the same tendency, which gives evidence that RRRP possesses good antioxidant capacity. The conclusion could be summed up that RRRP maintains excellent hepatoprotective effect and declines the mortality rate through cutting down the oxidative stress.

ALT, AST, and ALP are readily available, inexpensive, and routine biochemical assay used in clinical practice. Their activities are influenced by various factors, including viral hepatitis, alcohol consumption, and medication (Liu et al., 2014). Extremely high value of them reflects disease and inflammation of liver. The values of the RRRP group were lower than that of the VC group in all stages, concluding that RRRP could decline hepatic injury. The results of liver 17 
change, liver lesion scoring also concluded RRRP could decrease hepatic injury, which presented further proof of it.

In a word, RRRP could rise up the VIR, relieve liver pathological change, reduce the ducklings mortality, liver lesion scoring and oxidative stress. As oxidative damage is essential to hepatic injury, we did the job to find out the relationship between peroxidative injury evaluation indexes and hepatic injury evaluation indexes. Significant positive correlation between MDA and AST, ALP demonstrated that the high levels of AST, ALP had something to do with the rise of MDA content, proving that hepatic injury was induced by peroxidative injury in DVH to some degree.

Meanwhile, the correlation between oxidative injury evaluation indexes and mortality also declared that RRRP could down-regulate mortality by scavenging free radicals. To sum up, RRRP could significantly alleviate hepatic injury by releasing peroxidative injury, resulting in mortality descent.

\section{Conclusions}

RRRP had excellent anti-DHAV property that was related to alleviate hepatic injury especially peroxidative injury induced by DHAV. RRRP could be a crucial component in constructing new anti-DVH traditional Chinese herb formulat. The mechanism of RRRP against DHAV provided a platform to test novel drug strategies for hepatitis A virus in human beings.

\section{Acknowledgement}

The project was supported by National Natural Science Foundation of China (Grant No. 31172355, 31572557), the Project Funded by the Priority Academic Program Development of Jiangsu Higher Education Institutions (PAPD), the Special Fund for Agro-scientific Research in the Public Interest (201303040, 201403051). We are grateful to all other staff in the Institute of Traditional Chinese Veterinary Medicine of Nanjing Agricultural University for their assistances in the experiments.

\section{Conflict of interest}

We declare that we have no conflict of interest.

\section{References}

1. Abd Ellah, M.R., (2011). The role of liver biopsy in detection of hepatic oxidative stress. Vet Med Int, 2011:613602.

2. Adedara, I.A., Teberen, R., Ebokaiwe, A.P., Ehwerhemuepha, T., Farombi, E.O., (2012). Induction of oxidative stress in liver and kidney of rats exposed to Nigerian bonny light crude oil. Environ Toxicol, 27(6):372-379.

3. Bhopale, K.K., Wu, H., Boor, P.J., Popov, V.L., Ansari, G.A., Kaphalia, B.S., (2006). Metabolic basis of ethanol-induced hepatic and pancreatic injury in hepatic alcohol dehydrogenase deficient deer mice. Alcohol, 39(3):179-188.

4. Cardin, R., D'Errico, A., Fiorentino, M., Cecchetto, A., Naccarato, R., Farinati, F., (2002). Hepatocyte proliferation and apoptosis in relation to oxidative damage in alcohol-related liver disease. Alcohol Alcohol, 37(1):43-48.

5. Cardozo, F.T., Larsen, I.V., Carballo, E.V., Jose, G., Stern, R.A., Brummel, R.C., Camelini, C.M., Rossi, M.J., Simões, C.M., Brandt, C.R., (2013). In vivo anti-herpes simplex virus activity of a sulfated derivative of Agaricus brasiliensis mycelial polysaccharide. Antimicrob Agents Chemother, 57(6):2541-2549.

6. Chen, Y., Song, M., Wang, Y., Xiong, W., Zeng, L., Zhang, S., Xu, M., Du, H., Liu, J., Wang, D., et al., (2015). The anti-DHAV activities of Astragalus polysaccharide and its sulfate compared with those of BSRPS and its sulfate. 18 


\section{Song et al., Afr J Tradit Complement Altern Med., (2017) 14 (4): 8-21 \\ https://doi.org/10.21010/ajtcam.v14i4.2}

Carbohydr Polym, 117:339-345.

7. Chen, Y., Xiong, W., Zeng, L., Wang, D., Liu, J., Wu, Y., Hu, Y., (2014a). Comparison of Bush Sophora Root polysaccharide and its sulfate's anti-duck hepatitis A virus activity and mechanism. Carbohydr Polym, 102:333-340.

8. Chen, Y., Xiong, W., Zeng, L., Wang, Y., Zhang, S., Xu, M., Song, M., Du, H., Liu, J., Wang, D., et al., (2014b). Bush Sophora Root polysaccharide and its sulfate can scavenge free radicals resulted from duck virus hepatitis. Int $\mathbf{J}$ Biol Macromol, 66:186-193

9. Cui, B., Chen, Y., Liu, S., Wang, J., Li, S., Wang, Q., Chen, M., Lin, X., (2012). Antitumour activity of Lycium chinensis polysaccharides in liver cancer rats. Int J Biol Macromol, 51(3):314-318.

10. Cumurcu, B.E., Ozyurt, H., Ates, O., Gul, I.G., Demir, S., Karlıdag, R., (2013). Analysis of manganese superoxide dismutase (MnSOD: Ala-9Val) and glutathione peroxidase (GSH-Px: Pro 197 Leu) gene polymorphisms in mood disorders. Bosn J Basic Med Sci, 13(2):109-113.

11. Demir, I., Kiymaz, N., Gudu, B.O., Turkoz, Y., Gul, M., Dogan, Z., Demirtas, S., (2013). Study of the neuroprotective effect of ginseng on superoxide dismutase (SOD) and glutathione peroxidase (GSH-Px) levels in experimental diffuse head trauma. Acta Neurochir (Wien), 155(5):913-922.

12. Emzhik, M., Rahimi-Moghaddam, P., Ebrahimi, S.A., Keyhanfar, F., Moazzam, A.S., (2015). Commentary on prevention a possible drug-drug interaction: is concurrent administration of orlistat and pioglitazone increase the risk of durg-induced hepatotoxicity? Int J Prev Med, 6:16.

13. Erfan, A.M., Selim, A.A., Moursi, M.K., Nasef, S.A., Abdelwhab, E.M., (2015). Epidemiology and molecular characterisation of duck hepatitis A virus from different duck breeds in Egypt. Vet Microbiol, 177(3-4):347-352.

14. Faccin-Galhardi, L.C., Yamamoto, K.A., Ray, S., Ray, B., Carvalho Linhares, R.E., Nozawa, C., (2012). The in vitro antiviral property of Azadirachta indica polysaccharides for poliovirus. J Ethnopharmacol, 142(1):86-90.

15. Fu, Y., Pan, M., Wang, X., Xu, Y., Yang, H., Zhang, D., (2008). Molecular detection and typing of duck hepatitis A virus directly from clinical specimens. Vet Microbiol, 131(3-4):247-257.

16. Gabriel, A., Kuddus, R.H., Rao, A.S., Watkins, W.D., Gandhi, C.R., (1998). Superoxide-induced changes in endothelin (ET) receptors in hepatic stellate cells. J Hepatol, 29(4):614-627.

17. García-Villalón, D., Gil-Fernández, C., (1991). Antiviral activity of sulfated polysaccharides against African swine fever virus. Antiviral Res, 15(2):139-148.

18. Guo, L., Liu, J., Hu, Y., Wang, D., Li, Z., Zhang, J., Qin, T., Liu, X., Liu, C., Zhao, X., et al., (2012). Astragalus polysaccharide and sulfated epimedium polysaccharide synergistically resist the immunosuppression. Carbohydr Polym, 90(2):1055-1060.

19. Heidari, R., Niknahad, H., Jamshidzadeh, A., Eghbal, M.A., Abdoli. N., (2015). An overview on the proposed mechanisms of antithyroid drugs-induced liver injury. Adv Pharm Bull, 5(1):1-11.

20. Henrion, J., (2012). Hypoxic hepatitis. Liver Int, 32(7):1039-1052.

21. He, Y., Liu, Q., Li, Y., Yang, X., Wang, W., Li, T., Zhang, W., Cui, Y., Wang, C., Lin, R., (2015). Protective effects of hydroxysafflor yellow A (HSYA) on alcohol-induced liver injury in rats. J Physiol Biochem, 71(1):69-78.

22. Huang, X.Y., Hu, Y.L., Lu, Y., Zhang, F., Guo, Z.H., (2008). Sulfated modification conditions optimization of astragalus polysaccharide by orthogonal test and anti-IBDV activity determination of the modifiers. Zhong Yao Cai, 31(4):588-592.

23. Hu, J., Han, H., Lau, M.Y., Lee, H., MacVeigh-Aloni, M., Ji, C., (2015). Effects of combined alcohol and anti-HIV drugs on cellular stress responses in primary hepatocytes and hepatic stellate and kupffer cells. Alcohol Clin Exp Res, 39(1):11-20.

24. Jacobson, G.A., Ives, S.J., Narkowicz, C., Jones, G., (2012). Plasma glutathione peroxidase (GSH-Px) concentration is elevated in rheumatoid arthritis: a case-control study. Clin Rheumatol, 31(11):1543-1547.

25. Kankofer, M., Wawrzykowski, J., Hoedemaker, M., (2013). The presence of SOD 1 and GSH-Px in bovine retained and 19 
properly released foetal membranes. Reprod Domest Anim, 48(4):699-704.

26. Khademi, H., Khozeimeh, F., Tavangar, A., Amini, S., Ghalayani, P., (2014). The Serum and salivary level of malondialdehyde, vitamins A, E, and C in patient with recurrent aphthous stomatitis. Adv Biomed Res, 3:246.

27. Kim, M.C., Kim, M.J., Kwon, Y.K., Lindberg, A.M., Joh, S.J., Kwon, H.M., Lee, Y.J., Kwon, J.H., (2009). Development of duck hepatitis A virus type 3 vaccine and its use to protect ducklings against infections. Vaccine, 27(48):6688-6694.

28. Kim, M.S., Kim, S.H., (2011). Inhibitory effect of astragalin on expression of lipopolysaccharide-induced inflammatory mediators through NF-kB in macrophages. Arch Pharm Res, 34(12):2101-2107.

29. Kim, S.H., Lee, J.H., Lee, B.H., Yoon, H.J., Shin, D.H., Park, S.S., Jang, S.B., Park, J.S., Jee, Y.K., (2015). Superoxide Dismutase Gene (SOD1, SOD2, and SOD3) Polymorphisms and Antituberculosis Drug-induced Hepatitis. Allergy Asthma Immunol Res, 7(1):88-91.

30. Lanford, R.E., Feng, Z., Chavez, D., Guerra, B., Brasky, K.M., Zhou, Y., Yamane, D., Perelson, A.S., Walker, C.M., Lemon, S.M., (2011). Acute hepatitis A virus infection is associated with a limited type I interferon response and persistence of intrahepatic viral RNA. Proc Natl Acad Sci U S A, 108(27):11223-11228.

31. Lee, I.H., Huang, R.L., Chen, C.T., Chen, H.C., Hsu, W.C., Lu, M.K., (2002). Antrodia camphorata polysaccharides exhibit anti-hepatitis B virus effects. FEMS Microbiol Lett, 209(1):63-67.

32. Li, F., Liang, D., Yang, Z., Wang, T., Wang, W., Song, X., Guo, M., Zhou, E., Li, D., Cao, Y., et al., (2013). Astragalin suppresses inflammatory responses via down-regulation of NF- $\kappa \mathrm{B}$ signaling pathway in lipopolysaccharide-induced mastitis in a murine model. Int Immunopharmacol, 17(2):478-482.

33. Li, F., Wang, W., Cao, Y., Liang, D., Zhang, W., Zhang, Z., Jiang, H., Guo, M., Zhang, N., (2014). Inhibitory effects of astragalin on lipopolysaccharide-induced inflammatory response in mouse mammary epithelial cells. J Surg Res, 192(2):573-581.

34. Li, S., Zhang, B., Jiang, D., Wei, Y., Zhang, N., (2010). Herb network construction and co-module analysis for uncovering the combination rule of traditional Chinese herbal formulae. BMC Bioinformatics, 11 Suppl 11:S6.

35. Liu, Z., Que, S., Xu, J., Peng, T., (2014). Alanine aminotransferase-old biomarker and new concept: a review. Int J Med Sci, 11(9):925-935.

36. Livrea, M.A., Tesoriere, L., Pintaudi, A.M., Calabrese, A., Maggio, A., Freisleben, H.J., D'Arpa, D., D'Anna, R., Bongiorno, A., (1996). Oxidative stress and antioxidant status in beta-thalassemia major: iron overload and depletion of lipid-soluble antioxidants. Blood, 88(9):3608-3614.

37. Luper, S., (1998). A review of plants used in the treatment of liver disease: part 1. Altern Med Rev, 3(6):410-421.

38. Nuong, N.M., Vy, N.T., Duong, H.H., (2014). Combinative effects of thanh hao miet giap thang (sweet wormwood and tortoise shell decoction) ingredients on antioxidative activity in vitro. Afr $\mathrm{J}$ Tradit Complement Altern Med, 11(4):136-141.

39. Olszewska, M.A., Michel, P., (2012). Activity-guided isolation and identification of free radical-scavenging components from various leaf extracts of Sorbus aria (L.) Crantz. Nat Prod Res, 26(3):243-254.

40. Selegean, M., Putz, M.V., Rugea, T., (2009). Effect of the polysaccharide extract from the edible mushroom Pleurotus ostreatus against infectious bursal disease virus. Int J Mol Sci, 10(8):3616-3634.

41. Shen, Y.C., Chou, C.J,, Wang, Y.H., Chen, C.F., Chou, Y.C., Lu, M.K., (2004). Anti-inflammatory activity of the extracts from mycelia of Antrodia camphorata cultured with water-soluble fractions from five different Cinnamomum species. FEMS Microbiol Lett, 231(1):137-143.

42. Shi, S., Chen, H., Chen, Z., Fu, G., Wan, C., Huang, Y., Lin, S., Cheng, L., Fu, Q., Lin, J. et al., (2013). Complete genome sequence of a duck hepatitis a virus 1 isolated from a pigeon in china. Genome Announc, 1(4):e00451-13.

43. Singh, M.P., Majumdar, M., Thapa, B.R., Gupta, P.K., Khurana, J., Budhathoki, B., Ratho, R.K., (2015). Molecular characterization of hepatitis A virus strains in a tertiary care health set up in north western India. Indian J Med Res, 20 
Song et al., Afr J Tradit Complement Altern Med., (2017) 14 (4): 8-21

https://doi.org/10.21010/ajtcam.v14i4.2

141(2):213-220.

44. Song, X., Yin, Z., Zhao, X., Cheng, A., Jia, R., Yuan, G., Xu, J., Fan, Q., Dai, S., Lu, H. et al., (2013). Antiviral activity of sulfated Chuanmingshen violaceum polysaccharide against Newcastle disease virus. J Gen Virol, 94(Pt 10):2164-2174.

45. Strick-Marchand, H., Dusséaux, M., Darche, S., Huntington, N.D., Legrand, N., Masse-Ranson, G., Corcuff, E., Ahodantin, J., Weijer, K., Spits, H. et al., (2015). A novel mouse model for stable engraftment of a human immune system and human hepatocytes. PLoS One, 10(3):e0119820.

46. Sui, Z., Li, L., Liu, B., Gu, T., Zhao, Z., Liu, C., Shi, C., Yang, R., (2013). Optimum conditions for Radix Rehmanniae polysaccharides by RSM and its antioxidant and immunity activity in UVB mice. Carbohydr Polym, 92(1):283-288.

47. Triassi, M., Ottaiano, E., Diana, M.V., Pennino, F., Avolio, S., Dattilo, W., Simonetti, A., (2012). Epidemiologic situation of viral hepatitis type A in Campania: perspectives of prevention and control of infections at the local level. Ann Ig, 24(1):73-80.

48. Wang, Y., Li, Y., Liu, Y., Chen, X., Wei, X., (2015). Extraction, characterization and antioxidant activities of Se-enriched tea polysaccharides. Int J Biol Macromol, 77:76-84.

49. Wei, C.Y., Su, S., Huang, Z., Zhu, W.J., Chen, J.D., Zhao, F.R., Wang, Y.J., Xie, J.X., Wang, H., Zhang, G., (2012). Complete genome sequence of a novel duck hepatitis A virus discovered in southern China. J Virol, 86(18):10247.

50. Wen, H., Han, L., Zhang, X., Lian, C., Zhao, L., Si, C., Chen, H., (2014). Duck hepatitis A virus (DHAV) genotype definition: comment on the article by Cha et al. Vet Microbiol, 170(3-4):462-464.

51. Xiong, W., Chen, Y., Wang, Y., Liu, J., (2014). Roles of the antioxidant properties of icariin and its phosphorylated derivative in the protection against duck virus hepatitis. BMC Vet Res, 10:226.

52. Zhu, W., Yang, B., Fu, H., Ma, L., Liu, T., Chai, R., Zheng, Z., Zhang, Q., Li, G., (2015). Flavone inhibits nitric oxide synthase (NOS) activity, nitric oxide production and protein S-nitrosylation in breast cancer cells. Biochem Biophys Res Commun, 458(3):590-595. 